\title{
No correlation between somatostatine 2 receptor expression analyzed by RNA in-situ hybridization and real-time qRT-PCR in clinically non-functioning pituitary adenomas
}

\author{
(2) \\ P 834 \\ Filip Gabalec', Ales Kohout2, Jan Laco², Monika Drastikova ${ }^{3},{ }^{2}$ Martin Beranek ${ }^{3}$, David Netuka ${ }^{4}$, \\ Vaclav Masopust ${ }^{4}$, Tomas Cesak ${ }^{5}$, Jan Cap ${ }^{1}$ \\ 14th Dept. of Internal Medicine, ${ }^{2}$ Fingerland's Dept. of Pathology, ${ }^{3}$ Institute of Clinical Biochemistry and Diagnostics and ${ }^{5}$ Dept. of \\ Neurosurgery, Charles University Hospital and Faculty of Medicine in Hradec Kralove, Hradec Kralove; ${ }^{4}$ Dept. of Neurosurgery, Central \\ Military Hospital and $1^{\text {st }}$ Faculty of Medicine, Charles University, Prague, Czech Republic
}

\section{Objective}

The aim of this study was to quantitatively estimate somatostatine 2 receptor (SSTR2) in clinically non-functioning pituitary adenomas (CNFAs) with use of RNA in-situ hybridization (ISH) and quantitative real-time RT-PCR and correlate the results of both methods.

\section{Methods}

A standard histological and immunohistochemical examination was performed on the resected pituitary tumour including quantitative real-time RT-PCR as described previously. (Gabalec et al., Pituitary, 2012)

ISH for SSTR2/mRNA was performed using the RNAscope ${ }^{8} 2.0$ FFPE Assay (Advanced Cell Diagnostics, Inc., Hayward, CA, USA) on 4- $\mu$ m formalin fixed and paraffin embedded tissue sections. After deparaffinization they were pretreated with heat and protease prior to hybridization with a target probe to the SSTR2. A horseradish peroxidase-based signal amplification system was then hybridized to the target probes followed by color development with 3,3'-diaminobenzidine. Control probes for the bacterial gene DapB (negative control) and for the POLR2A gene (positive control - evidence of adequate RNA) were also included in each case. The study slides (and corresponding controls) were read independently by two study pathologists (A.K. and J.L) and classified in a binary manner as either positive or negative. Positive cases had to have granular cytoplasmic and/or nuclear brown staining that was higher than the signal on the DapB negative controlslide. Positive cases were subsequently semi-quantitatively assessed according to the manufacturer's scoring guideline as follows:
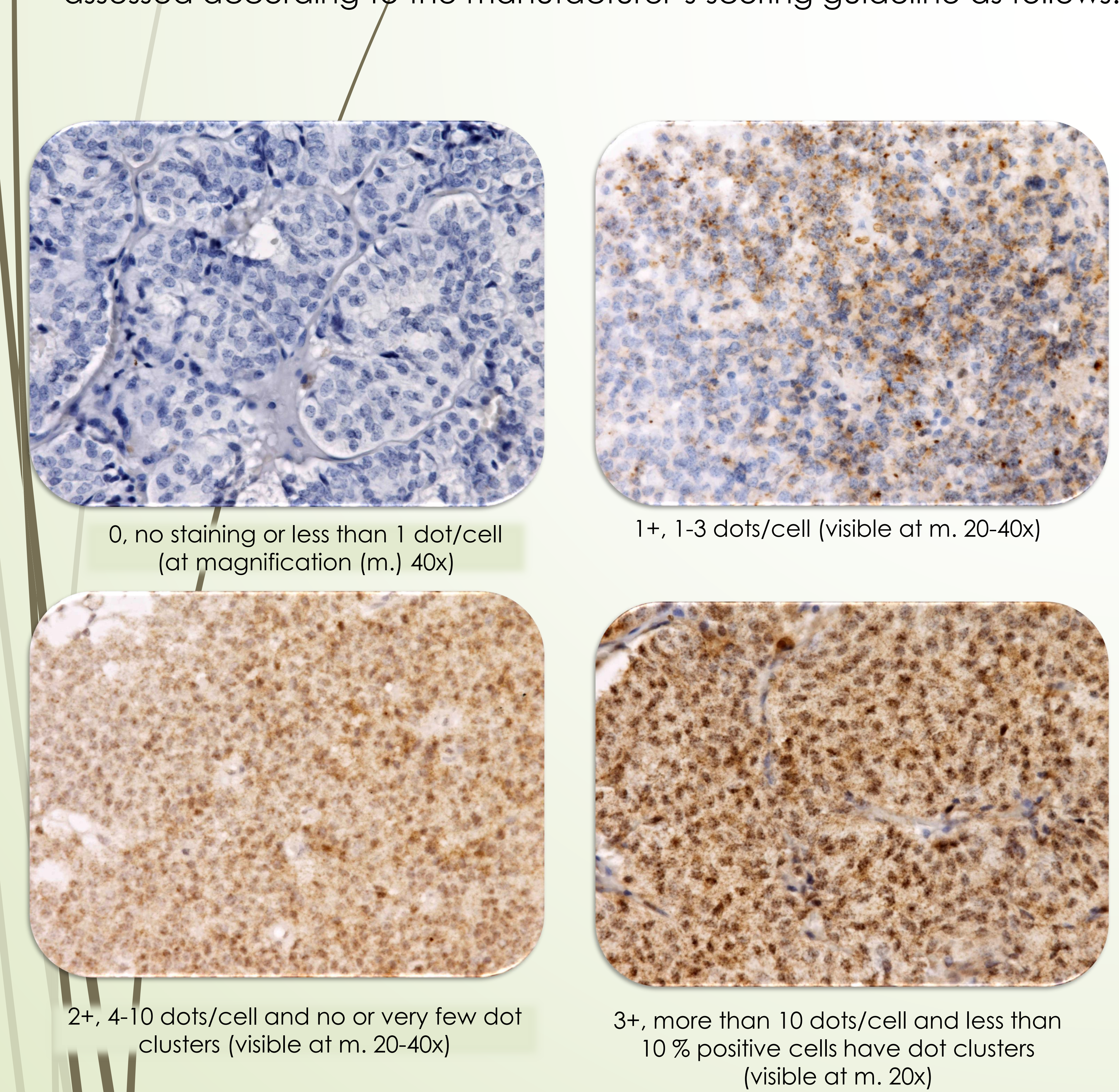

Results

25 adenomas with positive SSTR2 mRNA expression in qRT-PCR were chosen for further evaluation with RNA-ISH to compare both methods.

SSTR2 mRNA was expressed in all adenomas from 1413-148680 copies/5 $\mu$ l cDNA; the median of relative quantity (after normalization to housekeeping gene GUS) for SSTR2 was $111 \%$.

In contrast to GRT-PCR immunostaining was positive only in 9 adenomas with the use of RNA-ISH.

Positive cases were subsequently semi-quantitatively assessed according to the manufacturer's scoring guideline as follows: 1 adenoma with $1+, 5$ with $2+$ and 3 with $3+$. No adenoma scored 4+ although high expression of SSTR2 mRNA was present.

We did not find any correlation between data (Spearman's rank correlation coefficient 0,243 ).

(visible at m. 20x)

\section{Conclusion}

Use of somatostatine analogues or dopastatins remains controversial in CNFAs. Verification of SSTR presence before use of drug treatment should be useful. Both RNA ISH and qRT-PCR have their pitfalls.

In our case:

No correlation between these methods.

RNA-ISH is definitely less sensitive and specific.

We think that results for SSTR2 expression in RNA-ISH and QRT-PCR should be interpreted carefully. 